\title{
A new era for treatment of drug-resistant tuberculosis
}

\author{
Neel R. Gandhi ${ }^{1}$, James C.M. Brust ${ }^{2}$ and N. Sarita Shah ${ }^{3}$ \\ Affiliations: ${ }^{1}$ Rollins School of Public Health and Emory School of Medicine, Emory University, Atlanta, GA, \\ USA. ${ }^{2}$ Albert Einstein College of Medicine and Montefiore Medical Center, Bronx, NY, USA. ${ }^{3}$ Division of Global \\ HIV and Tuberculosis, US Centers for Disease Control and Prevention, Atlanta, GA, USA.
}

Correspondence: Neel R. Gandhi, Rollins School of Public Health, Emory University, 1518 Clifton Rd NE, CNR 3031, Atlanta, GA 30322, USA. E-mail: neel.r.gandhi@emory.edu

@ERSpublications

Adoption of new treatment regimens for drug-resistant TB has been slowed amid concerns regarding their efficacy and safety. Numerous studies worldwide now provide substantial evidence to support widespread implementation of these new drugs and regimens. http://ow.ly/FX8I30lpANX

Cite this article as: Gandhi NR, Brust JCM, Shah NS. A new era for treatment of drug-resistant tuberculosis. Eur Respir J 2018; 52: 1801350 [https://doi.org/10.1183/13993003.01350-2018].

For three decades, drug-resistant tuberculosis (TB) has posed grave challenges to patients, communities and global TB control efforts. Treatment of multidrug-resistant (MDR)-TB (i.e. TB resistant to at least isoniazid and rifampicin) and extensively drug-resistant (XDR)-TB (i.e. MDR-TB with additional resistance to fluoroquinolones and second-line injectable TB drugs) has relied on medications that are less potent and more toxic than first-line TB therapy. Consequently, drug-resistant TB treatment takes 2 years to complete and has been associated with frequent and severe side-effects. Treatment outcomes remain abysmal despite this prolonged therapy, with only $54 \%$ of MDR-TB patients and $30 \%$ of XDR-TB patients achieving cure [1]. When considering the full cascade of individuals estimated to have drug-resistant TB, only $12 \%$ of MDR-TB and $6.8 \%$ of XDR-TB patients are cured (figure 1).

One of the most effective drugs used for drug-resistant TB treatment is a medication class that must be given by injection for the first 6-8 months (i.e. kanamycin, amikacin and capreomycin) [2]. Health systems have struggled to create appropriate treatment programmes that can provide daily injections, often subjecting patients to prolonged hospitalisations for the duration of injectable therapy. These drugs are also associated with permanent hearing loss, leaving countless individuals worldwide profoundly impaired or deaf [3]. Other common severe adverse events from drug-resistant TB treatment include hypothyroidism, renal impairment and gastrointestinal intolerance $[4,5]$. From a patient perspective, this is further compounded by psychosocial, financial and personal hardships from the prolonged and toxic treatment course $[6,7]$.

There have been several key innovations in recent years that, together, have brought us to a tipping point in revolutionising the care of patients with MDR- and XDR-TB. In 2012, bedaquiline, the first new TB medication in more than 40 years, was approved by the US Food and Drug Administration (FDA) following successful treatment of MDR-TB patients in a phase 2B study [8]. Approximately 6 months later, delamanid, in yet another new drug class, was approved by the European Medicines Agency [9]. Linezolid (developed to treat resistant Gram-positive bacterial infections in the 1990s) was shown to have efficacy against TB, while clofazimine, a medication used for decades to treat leprosy, was found to have more activity against TB than previously thought $[10,11]$. Another major innovation came in 2010 from observational data among MDR-TB patients in Bangladesh, where clinician-scientists found that using a 

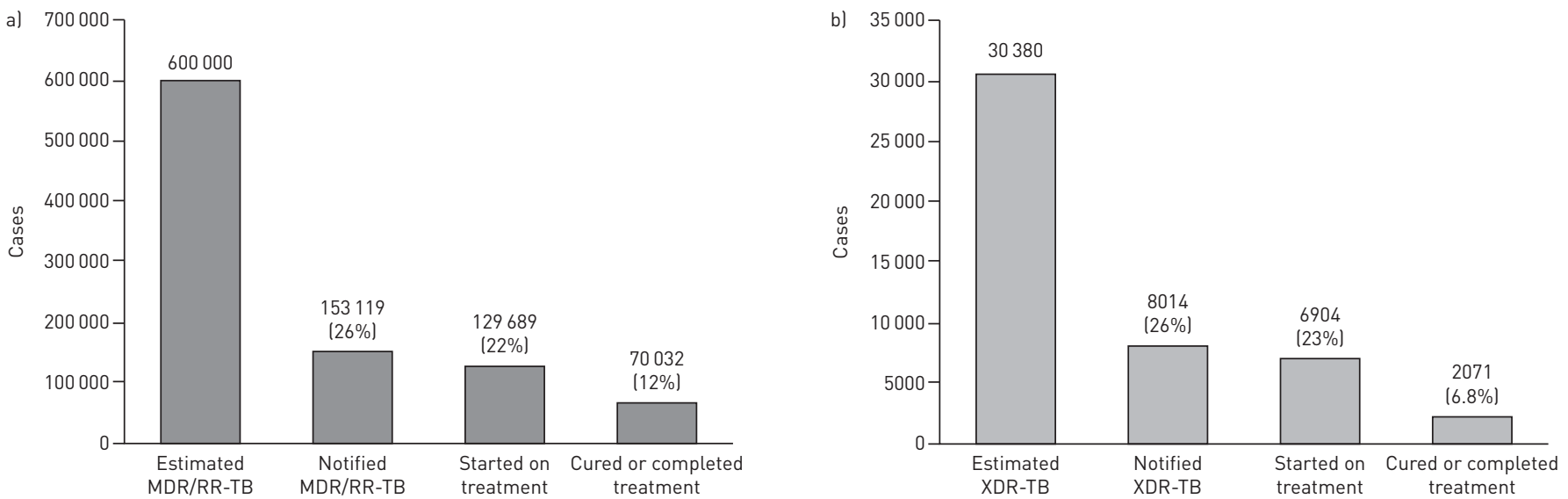

FIGURE 1 Treatment cascade. a) 12\% of estimated multidrug-resistant (MDR) or rifampicin-resistant (RR) tuberculosis (TB) cases and b) $6.8 \%$ of estimated extensively drug-resistant (XDR)-TB cases are diagnosed, initiated on appropriate treatment and cured of their drug-resistant TB disease. Data from [1].

new combination of medications could reduce MDR-TB treatment duration from 18-24 months to 9 months, while maintaining similar cure rates [12].

Despite these advances, various concerns have slowed the adoption of new medications and regimens into clinical practice and public health programmes. In the initial trial, bedaquiline was associated with a higher number of unexplained deaths compared with placebo. These deaths, along with the risk of QT-interval prolongation from bedaquiline, resulted in a black box warning by the FDA. Delamanid and a few other medications used for drug-resistant TB also prolong the QT-interval; this has both discouraged use of bedaquiline and delamanid, as well as creating reservations about combining them together or with other effective drugs, such as moxifloxacin. Additional barriers for uptake of bedaquiline and delamanid include high cost and slow progress in registering these medicines in high-burden countries. Consequently, roll-out has been sluggish and only a small fraction $(<5 \%)$ of patients in need of these drugs have actually received them [13]. Similarly, uptake of the short-course (i.e. 9-month) regimen was initially slow for a number of reasons. Because the regimen was discovered in an observational and operational nature without a comparator arm, there were concerns that it may not be effective in a diversity of settings, patient populations or Mycobacterium tuberculosis strains [14].

Recent data from cohorts worldwide, however, provide substantial evidence that we can move beyond these concerns. Although each study or report in isolation may not be considered definitive, the breadth of the collective experience speaks to the effectiveness and safety of these new drugs and regimens. In early 2017, a presentation at the Conference on Retroviruses and Opportunistic Infections indicated the tide was turning [15]. The Global Alliance for TB Drug Development reported the preliminary findings of the Nix TB study, a clinical trial evaluating a novel regimen of bedaquiline, linezolid and pretomanid (an experimental drug in the same class as delamanid) lasting only 6-9 months for the treatment of XDR-TB (in contrast to the recommended 24 months). Despite the high degree of drug resistance, investigators found that $36(90 \%)$ out of 40 participants who completed this 6-month regimen converted their TB cultures from positive to negative. Moreover, among the 31 patients who completed 6 months of post-treatment follow-up, 29 (94\%) remained cured and relapse free. This groundbreaking study demonstrated that even the most resistant forms of TB can be cured in a dramatically shorter time using a regimen containing new $\mathrm{TB}$ drugs. There have also been numerous reports of treatment using combinations of bedaquiline, linezolid, delamanid and clofazimine added to traditional drug-resistant TB medications, providing data from thousands of patients [16-23]. Patients with pre-XDR-TB (MDR-TB plus resistance to either the fluoroquinolones or injectables, but not both) and XDR-TB whose previous hope of cure was $<50 \%$ now have reported cure rates in excess of $60-80 \%$ from multiple settings [18].

Concerns regarding the safety of these medicines, whether using bedaquiline or delamanid individually or in combination with other QT-prolonging medicines, have also been allayed as global experience with their use increases [24]. Although mild and moderate adverse events remain common, few additional severe adverse events have been seen when these drugs are added to the existing background regimens [17, 20, 25]. Severe prolongation of the QT-interval also remains uncommon; even when it occurs, it appears to be reversible with cessation of therapy $[19,24,25]$. While the majority of these data are from use of bedaquiline or delamanid alone (with a standard MDR-TB regimen), early data on combined use of these drugs has shown co-treatment to be safe and well-tolerated, especially when compared to other 
MDR/XDR-TB regimens [26]. Furthermore, the mortality benefit gained from effectively treating MDR-, pre-XDR- and XDR-TB seems to outweigh the risk of death noted in the original trial [27].

Another breakthrough came in October 2017 when scientists at the Union World Conference on Lung Health reported phase 3 data from a randomised trial evaluating the shorter regimen for MDR-TB treatment. The STREAM (Standardised Treatment Regimen of Anti-tuberculosis Drugs for Patients with MDR-TB) trial was conducted in four countries and compared a modified short-course MDR-regimen to the standard 24-month World Health Organization (WHO) regimen [28]. The trial demonstrated high rates of cure $(78.1 \%)$ in the 9-month study arm. Although the trial did not demonstrate noninferiority compared to the 24-month regimen, this was due in part to a surprisingly high rate of favourable outcomes $(80.6 \%)$ in the control arm. Moreover, the shorter regimen costs less and fewer participants in that arm were lost to follow-up, suggesting it may be preferred by TB programmes and patients. These trial data bolster favourable effectiveness data from several observational studies comparing the 9-month treatment regimen to the conventional 24-month regimen [29]. Indeed, the WHO, which had previously endorsed the use of shorter regimens based on the observational studies, reaffirmed its recommendation following release of the STREAM trial data [30].

It has been just over a decade since the first report of XDR-TB galvanised the global TB community in their efforts to improve diagnosis and treatment of drug-resistant TB. 2017 and 2018 mark a turning point in this fight. With the breadth of data now available, it is time to implement these innovations for treating MDR- and XDR-TB. The WHO has been bold in creating guidelines encouraging the use of bedaquiline, delamanid and the 9-month shortened MDR-TB regimen [2]. Given the existing evidence, it is important that national drug-resistant TB treatment programmes now consider implementation of the 9-month regimen for patients with MDR-TB who have not been previously treated with second-line TB drugs, and in whom resistance fluoroquinolones and second-line injectable agents has been ruled out. The shortened regimen represents a critical step forward to minimise hardships of MDR-TB treatment for patients and programmes, while maintaining excellent cure rates. For patients with pre-XDR-TB and XDR-TB, inclusion of some combination of bedaquiline, linezolid, delamanid and clofazimine, in addition to any remaining effective, traditional second-line drugs improves cure rates. Further data from clinical trials is forthcoming but given the evidence already available, no time should be lost in combating these deadly diseases. The tools are in our hands, and it is now up to the global TB community, including funders and policy makers, to get these drugs to the patients that need them.

Author contributions: N.R. Gandhi wrote first draft of manuscript. J.C.M. Brust and N.S. Shah contributed to the writing of the manuscript. All authors agree with the manuscripts results and conclusions, and meet the International Committee of Medical Journal Editors criteria for authorship.

Conflict of interest: None declared.

Disclaimer: The findings and conclusions in this manuscript are those of the authors and do not necessarily represent the official position of the Centers for Disease Control and Prevention or the US Department of Health and Human Services.

Support statement: We gratefully acknowledge funding from the US National Institutes of Health (grants K24AI114444, R01AI114304 and R01AI138646). Funding information for this article has been deposited with the Crossref Funder Registry.

\section{References}

1 World Health Organization. Global Tuberculosis Report 2017. Report No.: WHO/HTM/TB/2017.23. Geneva, World Health Organization, 2017.

2 Falzon D, Schunemann HJ, Harausz E, et al. World Health Organization treatment guidelines for drug-resistant tuberculosis, 2016 update. Eur Respir J 2017; 49: 1602308.

3 Reuter A, Tisile P, von Delft D, et al. The devil we know: is the use of injectable agents for the treatment of MDR-TB justified? Int J Tuberc Lung Dis 2017; 21: 1114-1126.

4 Satti H, Mafukidze A, Jooste PL, et al. High rate of hypothyroidism among patients treated for multidrug-resistant tuberculosis in Lesotho. Int J Tuberc Lung Dis 2012; 16: 468-472.

5 Bloss E, Kuksa L, Holtz TH, et al. Adverse events related to multidrug-resistant tuberculosis treatment, Latvia, 2000-2004. Int J Tuberc Lung Dis 2010: 14: 275-281.

6 Nathanson E, Nunn P, Uplekar M, et al. MDR tuberculosis - critical steps for prevention and control. $N$ Engl J Med 2010; 363: 1050-1058.

7 Daftary A, Padayatchi N, O'Donnell M. Preferential adherence to antiretroviral therapy over tuberculosis treatment: a qualitative study of drug-resistant TB/HIV co-infected patients in South Africa. Glob Public Health 2014; 9: 1107-1116.

8 Diacon AH, Pym A, Grobusch M, et al. The diarylquinoline TMC207 for multidrug-resistant tuberculosis. $N$ Engl J Med 2009; 360: 2397-2405. 
9 Gler MT, Skripconoka V, Sanchez-Garavito E, et al. Delamanid for multidrug-resistant pulmonary tuberculosis. N Engl J Med 2012; 366: 2151-2160.

10 Lee M, Lee J, Carroll MW, et al. Linezolid for treatment of chronic extensively drug-resistant tuberculosis. $N$ Engl J Med 2012; 367: 1508-1518.

11 O'Donnell MR, Padayatchi N, Metcalfe JZ. Elucidating the role of clofazimine for the treatment of tuberculosis. Int J Tuberc Lung Dis 2016; 20: 52-57.

12 Van DA, Maug AK, Salim MA, et al. Short, highly effective, and inexpensive standardized treatment of multidrug-resistant tuberculosis. Am J Respir Crit Care Med 2010; 182: 684-692.

13 Medecins Sans Frontieres. Four years and counting: Slow scale up of newer MDR-TB drugs covers less than 5\% in need. 2017. msfaccess.org/MDR-TB-issue-brief-2017 Date last accessed: January 31, 2018. Date last updated: May 2018

14 Sotgiu G, Tiberi S, D'Ambrosio L, et al. WHO recommendations on shorter treatment of multidrug-resistant tuberculosis. Lancet 2016; 387: 2486-2487.

15 Conradie F, Diacon AH, Everitt D, et al. The Nix-TB trial of Pretomanid, Bedaquiline and Linezolid to treat XDR-TB. Conference on Retroviruses and Opportunistic Infections (CROI) 2017. www.croiconference.org/sessions/ nix-tb-trial-pretomanid-bedaquiline-and-linezolid-treat-xdr-tb

16 Guglielmetti L, Hewison C, Avaliani Z, et al. Examples of bedaquiline introduction for the management of multidrug-resistant tuberculosis in five countries. Int J Tuberc Lung Dis 2017; 21: 167-174.

17 Mohr E, Hughes J, Reuter A, et al. Delamanid for rifampicin-resistant tuberculosis: a retrospective study from South Africa. Eur Respir J 2018; 51: 1800017.

18 Borisov SE, Dheda K, Enwerem M, et al. Effectiveness and safety of bedaquiline-containing regimens in the treatment of MDR- and XDR-TB: a multicentre study. Eur Respir J 2017; 49: 1700387.

19 Pontali E, Sotgiu G, Tiberi S, et al. Combined treatment of drug-resistant tuberculosis with bedaquiline and delamanid: a systematic review. Eur Respir J 2018; 52: 1800934.

20 Guglielmetti L, Barkane L, Le Du D, et al. Safety and efficacy of exposure to bedaquiline-delamanid in multidrug-resistant tuberculosis: a case series from France and Latvia. Eur Respir J 2018; 51: 1702550.

21 Dalcolmo M, Gayoso R, Sotgiu G, et al. Effectiveness and safety of clofazimine in multidrug-resistant tuberculosis: a nationwide report from Brazil. Eur Respir J 2017; 49: 1602445.

22 Olayanju O, Limberis J, Esmail A, et al. Long-term bedaquiline-related treatment outcomes in patients with extensively drug-resistant tuberculosis from South Africa. Eur Respir J 2018; 51: 1800544.

23 Sotgiu G, D’Ambrosio L, Centis R, et al. Carbapenems to treat multidrug and extensively drug-resistant tuberculosis: a systematic review. Int J Mol Sci 2016; 17: 373.

24 Pontali E, Sotgiu G, Tiberi S, et al. Cardiac safety of bedaquiline: a systematic and critical analysis of the evidence. Eur Respir J 2017; 50: 1700146.

25 World Health Organization. Report of the Guideline Development Group Meeting on the use of bedaquiline in the treatment of multidrug-resistant tuberculosis: a review of available evidence (2016). Geneva, World Health Organization, 2017.

26 Ferlazzo G, Mohr E, Laxmeshwar C, et al. Early safety and efficacy of the combination of bedaquiline and delamanid for the treatment of patients with drug-resistant tuberculosis in Armenia, India, and South Africa: a retrospective cohort study. Lancet Infect Dis 2018; 18: 536-544.

27 Schnippel K, Ndjeka N, Maartens G, et al. Effect of bedaquiline on mortality in South African patients with drug-resistant tuberculosis: a retrospective cohort study. Lancet Respir Med 2018; 6: 699-706.

28 The Union 2017. https://www.theunion.org/news-centre/news/stream-clinical-trial-results-provide-vital-insightinto-nine-month-treatment-regimen-for-multidrug-resistant-tuberculosis Date last updated: October 13, 2017; date last accessed: September 12, 2018.

29 Trebucq A, Schwoebel V, Kashongwe Z, et al. Treatment outcome with a short multidrug-resistant tuberculosis regimen in nine African countries. Int J Tuberc Lung Dis 2018; 22: 17-25.

30 World Health Organization. Response to results from the STREAM stage 1 randomised controlled trial for treatment of MDR TB using a standardised shorter regimen. 2017. www.who.int/tb/features_archive/stream stage1_MDRTB/en/ Date last accessed: May 17, 2018. 